\title{
EL ORGANISMO ESTATAL INSPECCIÓN DE TRABAJO Y SEGURIDAD SOCIAL. ALGO MÁS QUE UN LUGAR EN COMÚN
}

\author{
Rafael García Matos \\ Inspector de Trabajo y de la Seguridad Social \\ rgarciamatos@mitramiss.es
}

\section{INTRODUCCIÓN}

El Boletín Oficial del Estado del 7 de abril de 2018 publicó el Real Decreto $192 / 2018$, de 6 de abril, por el que se aprueban los estatutos del organismo autónomo Organismo Estatal Inspección de Trabajo y Seguridad Social (en adelante, OEITSS). Esta publicación, largo tiempo esperada, consolida la previsión de la Ley 23/2015, de 22 de julio, Ordenadora del Sistema de Inspección de Trabajo y Seguridad Social (en adelante, LOSITSS), de situar a la Inspección de Trabajo y Seguridad Social (en adelante, ITSS) en el ámbito de la Administración institucional y regular la estructura organizativa del organismo autónomo.

Esta presentación pretende trazar un esbozo de esta regulación destacando, en primer lugar, las motivaciones de la creación del organismo autónomo ya contenidas en la LOSITSS y, en segundo lugar, los aspectos novedosos del nuevo modelo organizativo respecto del anterior. También hay que advertir, ya desde el principio, que la regulación del modelo de inspección del Real Decreto 192/2018, de 6 de abril, contempla únicamente el continente o recipiente para la ITSS, que habrá de completarse con dotaciones suficientes de medios - de manera sostenida en el tiempoque permitan el mejor y más efectivo ejercicio de la función inspectora: es la función la que justifica al órgano y no al revés. En definitiva, para que sea verdaderamente efectiva y no un mero ejercicio de lampedusismo administrativo, si se permite la expresión (ya se sabe, cambiarlo todo para que todo siga igual), la reforma del modelo exige una voluntad continuada de los gobiernos de turno (del gobierno de la nación y de los gobiernos de las Comunidades Autónomas) que garantice los medios para que la ITSS 
pueda realizar con eficacia sus cometidos al margen de los vaivenes políticos o de las eventuales coyunturas.

\section{EL NUEVO MODELO ORGANIZATIVO DE LA LOSITSS. PRINCIPALES MOTIVOS DE LA REFORMA}

Es la LOSITSS la norma que configura el nuevo modelo para la Inspección de Trabajo y Seguridad Social en España, adaptado al desdoblamiento de las Administraciones responsables de la ejecución de la legislación laboral en materia inspectora con el objeto principal de mejorar el servicio que se presta a los ciudadanos, evitando disfunciones y duplicidades.

El primer elemento ha de ponerse en conexión con el sistema constitucional de reparto de competencias entre el Estado y las Comunidades Autónomas en la ejecución de la legislación del orden social. La ejecución de la legislación laboral corresponde a las Comunidades Autónomas de acuerdo con el art. 149.1.7. ${ }^{\circ} \mathrm{CE}$, mientras que la gestión del régimen económico de la Seguridad Social es competencia del Estado (art. 149.1.18.a). La función inspectora abarca ambas materias, pero esta función no se despliega por separado, sino de manera conjunta e integrada, porque, como todo el mundo comprende, la realidad laboral abarca de manera inextricable los diversos aspectos o facetas que la componen. Precisamente por eso, la regulación anterior contenida en la Ley 42/1997 había previsto unos mecanismos de participación adecuados de las Comunidades Autónomas en el Sistema de Inspección desde el mantenimiento de la unidad organizativa del mismo, lo que implicaba la adscripción orgánica de todos los funcionarios del Sistema a la Administración General del Estado, sin perjuicio de la dependencia funcional de la Administración correspondiente que fuera titular de la materia objeto de la actuación inspectora.

No obstante, este modelo de participación se vio superado por la evolución en el ejercicio de sus competencias por parte de las Comunidades Autónomas.

Varios Estatutos de Autonomía revisados en la primera década de este siglo abordaron de manera prolija la cuestión de las competencias autonómicas en materia de Inspección de Trabajo y Seguridad Social.

En efecto, el art. 170.2 de la Ley Orgánica 6/2006, de 19 de julio, de reforma del Estatuto de Autonomía de Cataluña, señala que: «Corresponde a la Generalitat la competencia ejecutiva sobre la función pública ins- 
pectora en todo lo previsto en este artículo. A tal efecto, los funcionarios de los cuerpos que realicen dicha función dependerán orgánica y funcionalmente de la Generalitat. A través de los mecanismos de cooperación previstos en el título $\mathrm{V}$ se establecerán las fórmulas de garantía del ejercicio eficaz de la función inspectora en el ámbito social».

En iguales términos que el Estatuto de Autonomía de Cataluña se pronuncian los Estatutos correspondientes a Andalucía y Aragón, mientras que los Estatutos de la Comunidad Valenciana o de la Junta de Castilla y León contienen cláusulas dirigidas a prever un mayor nivel de autogobierno que podría llevar a la transferencia de efectivos del Sistema de Inspección a estas Comunidades.

En el año 2010, mediante el Real Decreto 206/2010, de 26 de febrero, se produjo el traspaso de funciones y servicios a la Generalitat de Cataluña en materia de función pública inspectora de la Inspección de Trabajo y Seguridad Social.

En el año 2011, mediante el Real Decreto 895/2011, de 24 de junio, se acordó el traspaso de funciones y servicios a la Comunidad Autónoma del País Vasco en materia de función pública inspectora de la Inspección de Trabajo y Seguridad Social.

La idea clave de la LOSITSS para evitar la disgregación y ruptura de la Inspección de Trabajo y Seguridad Social consiste en afianzar la unidad del Sistema de Inspección ${ }^{1}$, declarando como uno de sus principios ordenadores el de concepción única e integral del sistema, garantizándose su funcionamiento cohesionado mediante la coordinación, cooperación y participación de las diferentes Administraciones Públicas [art. 2.b) LOSITSS]. Por tanto, los servicios transferidos a las Comunidades Autónomas de Cataluña y el País Vasco forman parte del Sistema de Inspección de Trabajo y Seguridad Social ${ }^{2}$. Además de ello, la LOSITSS crea un organismo autó-

${ }^{1}$ Art. 1.1 LOSITSS: «1. El Sistema de Inspección de Trabajo y Seguridad Social está constituido por el conjunto de principios legales, normas, órganos, personal y medios materiales, incluidos los informáticos, que contribuyen al adecuado cumplimiento de la misión que tiene encomendada, según lo establecido en la presente Ley».

2 Exposición de Motivos, II, LOSITSS: «El Sistema se integra no solo por las Administraciones Públicas titulares de las competencias materiales de ejecución de la legislación laboral y de Seguridad Social, sino también por los órganos y las estructuras institucionales creadas para la coordinación de dichos órganos, entre las que se incluyen el Consorcio de la Inspección de Trabajo y Seguridad Social de Cataluña y el Consejo Vasco de Inspección de Trabajo y Seguridad Social». Vid. también el Convenio de colaboración con la Administración General de la Comunidad Autónoma del País Vasco y la Generalidad de Cataluña por el que se crea y regula la Comisión de Cooperación Interadministrativa del sistema de la Inspección de Trabajo y Seguridad Social (BOE de 16 de febrero de 2017).

Foro, Nueva época, vol. 21, núm. 1 (2018): 253-278 
nomo de participación conjunta de la Administración General del Estado y las Comunidades Autónomas, es decir, un órgano que preste servicio a cada una de las Administraciones en función de las materias que resulten de la competencia de una o de otras, de acuerdo con el orden constitucional de distribución de competencias.

Estos elementos básicos, el mantenimiento de la unidad del Sistema de Inspección y la autonomía del Organismo, junto con la participación en su órgano de dirección de máximo nivel, el Consejo Rector, de representantes paritarios de las Comunidades Autónomas y de la Administración General del Estado, permiten hablar de un modelo de Inspección compartido entre las distintas Administraciones Públicas con competencias en las materias que son objeto de atención por parte de la Inspección de Trabajo y Seguridad Social. A partir de este nuevo modelo organizativo puede decirse que la Inspección, en cuando órgano que despliega una función pública, es de todos y no se ejerce de prestado, como podría parecer con anterioridad, en la medida en que los funcionarios del Sistema de Inspección dependerán orgánicamente de un organismo autónomo participado conjuntamente por Estado y Comunidades Autónomas, manteniendo la tradicional dependencia funcional de una u otra Administración según la materia objeto de actuación inspectora.

Volviendo la oración por pasiva — también conviene señalarlo-, la Inspección, en cuanto función pública consistente en el ejercicio de la supervisión y control unificado de toda la normativa del orden social, no es de nadie. Y no es de nadie porque la función inspectora exige ejercerse con independencia, como quiere la propia LOSITSS (art. 8), en consonancia con los postulados de los Convenios 81 y 129 de la Organización Internacional del Trabajo, con sujeción plena, naturalmente, a la Ley y al Derecho (art. 9.3 de la Constitución Española), pero al margen de presiones o influencias indebidas provenientes de instancias de cualquier clase, entre las que pueden encontrarse, cómo no reconocerlo, las políticas.

La creación del organismo autónomo para la ITSS ya se hizo por la LOSITSS, en la medida en que la entonces vigente Ley 6/1997, de 14 de abril, de Organización y Funcionamiento de la Administración General del Estado (LOFAGE), que permitía y justificaba la creación de organismos públicos, exigía que ello se hiciera mediante ley (art. 63.1). Es la actual Ley 40/2015, de 1 de octubre, de Régimen Jurídico del Sector Público (que sustituyó a la LOFAGE), la que contiene la regulación actual de los organismos autónomos, por lo que el art. 1.1 de los Estatutos del OEITSS se refiere al art. 84.1.a) de dicha Ley para definirlo. 
Con ello el OEITSS, como señala el art. 1.2 de sus Estatutos, «tiene personalidad jurídica pública diferenciada, patrimonio y tesorería propios, así como autonomía de gestión y plena capacidad jurídica y de obrar», lo que le permite realizar, en régimen de descentralización funcional, la ejecución de programas específicos tanto en materias dependientes de la Administración General del Estado como de las Comunidades Autónomas para la más eficaz gestión del servicio público que tiene encomendado la Inspección, sin necesidad de que cada una de las Administraciones cuente con su propio Servicio de Inspección. Se posibilita con ello, además, el mantenimiento de la unidad de criterio en la interpretación de la norma y en la actuación inspectora en todo el territorio nacional, en aplicación de los principios de igualdad de trato y no discriminación en el ejercicio de la actividad inspectora de la Inspección de Trabajo y Seguridad Social (art. 20.2 LOSITSS), y se evita la doble visita de Inspección.

Es verdad que el modelo de Inspección diseñado por la LOSITSS y culminado por el Real Decreto 192/2018 no es enteramente simétrico, dado que dos Comunidades Autónomas (Cataluña y el País Vasco) cuentan con Servicios de Inspección fruto de los acuerdos antes referidos, lo que hace que en estas Comunidades Autónomas coexistan los servicios dependientes de las Comunidades Autónomas con los que mantiene la Administración General del Estado. Por eso la LOSITSS se esfuerza en reencauzar la gestión del Sistema como un todo unitario no solo mediante la declaración del principio de la unidad del Sistema, sino también estableciendo mecanismos efectivos para lograrlo ${ }^{3}$.

Como decíamos, fue la LOSITSS la creadora del OEITSS, pero es el Real Decreto 192/2018, que aprueba sus Estatutos, el que pone el mismo en funcionamiento, cumpliendo así la previsión establecida en la disposición adicional primera de la LOSITSS ${ }^{4}$. La entrada en funcionamiento

3 Disposición adicional octava LOSITSS, «Comunidades Autónomas con funciones y servicios traspasados en materia de función pública inspectora». Participación de estas Comunidades Autónomas en el Consejo Rector del OEITSS y creación de un órgano multilateral específico en los ámbitos materiales concretos que les sean de interés, en especial los criterios de coordinación de actuaciones supraautonómicas o que requieran una respuesta uniforme en todo el territorio nacional. Vid. Resolución de 6 de febrero de 2017 de la Secretaría General Técnica, la cual publica el Convenio de colaboración con la Administración General de la Comunidad Autónoma del País Vasco y la Generalidad de Cataluña por el que se crea y regula la Comisión de Cooperación Interadministrativa del Sistema de la Inspección de Trabajo y Seguridad Social (BOE de 16 de febrero de 2017).

${ }^{4}$ Disposición adicional primera LOSITSS: «1. La entrada en funcionamiento efectivo del Organismo Estatal Inspección de Trabajo y Seguridad Social tendrá lugar en la fecha establecida en sus Estatutos, una vez aprobados y publicado estos».

Foro, Nueva época, vol. 21, núm. 1 (2018): 253-278 
efectivo del OEITSS se produjo, por tanto, en fecha 8 de abril de 2018, de acuerdo con lo señalado en la disposición adicional primera del Real Decreto 192/2018 . Teniendo en cuenta esta fecha de entrada en funcionamiento, el OEITSS nace sin previsión presupuestaria propia, por lo que hasta tanto se apruebe el presupuesto del OEITSS, integrado en los Presupuestos Generales del Estado, los créditos presupuestarios para financiar las actuaciones de la Inspección de Trabajo se seguirán gestionando por los servicios hasta ahora competentes del Ministerio de Trabajo, Migraciones y Seguridad Social, y el pago del personal al servicio del Organismo estatal se efectuará con cargo a los créditos de procedencia (disposición transitoria primera del Real Decreto 192/2018). Habrá que esperar, por tanto, un poco más para verificar la verdadera puesta en marcha del OEITSS, lo que sucederá cuando disponga de un presupuesto propio y un patrimonio y tesorería también propios, que son condiciones necesarias (aunque no suficientes) para alcanzar la autonomía de gestión predicable de cualquier organismo autónomo.

\section{LA ESTRUCTURA DEL OEITSS}

Los capítulos II, III y IV de los Estatutos del OEITSS regulan los órganos de dirección y gobierno y participación institucional del OEITSS, y la estructura administrativa central y la estructura administrativa territorial, respectivamente. Este modelo organizativo es tributario - forzoso es decirlo- del modelo anterior, pero tiene la virtud, a mi juicio, de afianzar sus fortalezas y reducir en gran medida las debilidades que lo ponían en cuestión y fueron, al final, las que justificaron su reforma.

Con sus peculiaridades, la estructura del OEITSS responde a un modelo clásico de organismo autónomo. En una primera línea se sitúan el Consejo Rector, órgano de gobierno colegiado cuya singularidad radica en su composición paritaria de miembros de las Comunidades Autónomas y de la Administración General del Estado; un órgano de dirección, el director,

\footnotetext{
5 Real Decreto 192/2018: «Disposición adicional primera. Fecha de constitución del organismo autónomo Organismo Estatal Inspección de Trabajo y Seguridad Social:

1. El organismo autónomo Organismo Estatal Inspección de Trabajo y Seguridad Social entrará en funcionamiento efectivo y quedará constituido el día siguiente al de la publicación en el Boletín Oficial del Estado del presente Real Decreto.

2. Desde la fecha indicada en la disposición transitoria primera, el Organismo Estatal se subrogará en todos los derechos y obligaciones derivados de competencias de la Dirección General de Inspección de Trabajo y Seguridad Social atribuidas a esta».
} 
y un órgano de participación institucional, el Consejo General, compuesto también paritariamente de miembros del Consejo Rector y de los sindicatos y organizaciones empresariales que tienen la consideración de más representativos. La segunda línea está compuesta por la estructura administrativa central, el núcleo operativo del OEITSS, formada por unidades a cuyo frente está un funcionario con rango de subdirector general. Y la tercera línea está compuesta por la estructura administrativa territorial dedicada al ejercicio de la función inspectora como tal. Repasaremos a continuación la regulación de estas unidades destacando los aspectos más relevantes o novedosos que contiene.

\section{1. Órganos de dirección y de gobierno y de participación institucional del organismo autónomo Organismo Estatal}

\section{A. El Consejo Rector}

El Consejo Rector del OEITSS es el órgano superior de gobierno del Organismo. Se trata de un órgano colegiado formado por representantes de la Administración General del Estado y de las Comunidades Autónomas (art. 4.1 de los Estatutos). Que sea un órgano colegiado compuesto por representantes de distintas Administraciones Públicas y, al mismo tiempo, un órgano denominado rector es, como ya se ha indicado anteriormente, la expresión de la voluntad política de hacer de la Inspección de Trabajo y Seguridad Social una administración unificada en materia de función pública inspectora.

El Consejo Rector tiene su precedente inmediato en la Conferencia Sectorial de Asuntos Laborales en materia de Inspección de Trabajo y Seguridad Social, cuyos asuntos se sustanciaban en la Comisión de Trabajo de dicha Conferencia en los términos de los derogados ${ }^{6}$ arts. 42 y 43 del Reglamento de Organización y Funcionamiento de la Inspección de Trabajo y Seguridad Social aprobado por el Real Decreto 138/2000, de 4 de febrero.

EL Consejo Rector se compone de un presidente, que será el titular del Ministerio de Trabajo, Migraciones y Seguridad Social —o sea, el ministro/a - lo que refleja también la voluntad de otorgar al Consejo Rector el máximo nivel político; diecisiete vocales en representación de cada una de las Comunidades Autónomas, y dieciséis vocales en representación

${ }^{6}$ Disposición derogatoria única del Real Decreto 192/2018, de 6 de abril. 
de la Administración General del Estado con rango mínimo de director general (con el titular del Ministerio alcanzan diecisiete, logrando con ello la paridad). La designación de los miembros del Consejo Rector se hace por parte del Ministerio de Trabajo, Migraciones y Seguridad Social, si bien los de las Comunidades Autónomas se hace a propuesta vinculante de estas. Se regula también la distribución de los miembros del Consejo Rector por el cupo de la Administración General del Estado, indicando que nueve de ellos se propondrán por el Ministerio de Trabajo, Migraciones y Seguridad Social, por lo que otros siete lo serán por otros Ministerios ${ }^{7}$. Que existan representantes de otros Ministerios en el Consejo Rector del OEITSS refleja la transversalidad de la actuación de la Inspección de Trabajo y Seguridad Social y la necesidad de que se preste la debida atención a su funcionamiento en otros órdenes de actuación administrativa no estrictamente coincidentes, pero conexos, con los propios del orden social.

Es de ver, por otro lado, que por las Comunidades Autónomas hay un representante por cada una de ellas, totalizando diecisiete, es decir, que también se incluye en el Consejo Rector a representantes de las Comunidades Autónomas de Cataluña y el País Vasco, aun cuando estas tienen servicios de Inspección propios fruto de los acuerdos de 2010 y 2011, respectivamente, de traspaso de servicios en materia de función pública inspectora. La razón de la presencia de estas Comunidades Autónomas en el Consejo Rector del Organismo obedece al mandato de la propia LOSITSS de mantenimiento de la concepción única e integral del Sistema y específicamente a lo establecido en su art. $25^{8}$.

7 Uno por el Ministerio de Sanidad, Servicios Sociales e Igualdad; dos por el Ministerio de Hacienda y Función Pública; uno por el Ministerio del Interior; uno por el Ministerio de Economía, Industria y Competitividad; uno por el Ministerio de Justicia, y uno por el Ministerio de la Presidencia y para las Administraciones Territoriales [art. 5.1.c) Estatutos].

8 «Art. 25. Cooperación y participación de las Administraciones Públicas.

1. La Administración General del Estado y las Administraciones de las Comunidades Autónomas, en el ámbito de sus respectivas competencias, garantizarán el ejercicio y la eficacia del servicio público de la Inspección de Trabajo y Seguridad Social. Para ello, dichas Administraciones organizarán la realización de las actuaciones inspectoras con sujeción a los principios establecidos en el art. 2 y desarrollarán el principio de cooperación a través de los órganos e instrumentos previstos en esta Ley y en los acuerdos o convenios suscritos entre las Administraciones competentes.

2. La cooperación entre las Administraciones citadas en el apartado anterior se instrumentará mediante convenios de colaboración según lo establecido en el art. 6 de la Ley 30/1992, de 26 de noviembre.

3. Las Comunidades Autónomas participarán, asimismo, en la dirección del Sistema de Inspección de Trabajo y Seguridad Social a través del Consejo Rector del Organismo Estatal Inspección de Trabajo y Seguridad Social. 
Las funciones asignadas al Consejo Rector corresponden a las propias de los órganos de estas características. Son funciones de propuesta, impulso y aprobación de las decisiones de mayor trascendencia para la vida del Organismo. Ya se recogen en la LOSITSS y se amplían en los Estatutos del OEITSS 9,10 .

4. Con el fin de garantizar el funcionamiento cohesionado de la Inspección de Trabajo y Seguridad Social, la Administración General del Estado y la de las Comunidades Autónomas establecerán la realización de los planes y programas de actuación que sean necesarios para el logro de objetivos de carácter general.

Con la misma finalidad, las Administraciones competentes dispondrán de un sistema de información dirigido a garantizar la eficacia de la función inspectora y la coherencia y homogeneidad de los datos y de su tratamiento, de acuerdo con lo previsto en el art. 24».

9 «Art. 6. Funciones del Consejo Rector.

Corresponde al Consejo Rector:

a) Conocer y aprobar los planes y programas generales de actuación de la Inspección de Trabajo y Seguridad Social y las medidas y estrategias necesarias para su ejecución, y conocer los planes y programas territoriales de actuación.

b) Establecer e impulsar medidas de colaboración con otras Administraciones para el mejor ejercicio de las funciones encomendadas al Organismo Estatal.

c) Aprobar la propuesta del presupuesto de ingresos y gastos del Organismo Estatal y ser informado de su ejecución, así como aprobar las cuentas anuales.

d) Conocer e informar las propuestas normativas relativas al Sistema de Inspección de Trabajo y Seguridad Social, así como los criterios técnicos y procedimientos operativos comunes para el desarrollo de la función inspectora.

e) Adoptar propuestas en relación con la posición estatal en materia de Inspección de Trabajo y Seguridad Social en el ámbito europeo y la ejecución de las acciones y programas que impulse la Unión Europea y la cooperación administrativa transnacional.

f) Adoptar propuestas sobre las cuestiones que se le sometan en relación con la regulación y promoción de la carrera profesional del personal inspector, los procesos de ingreso, selección, formación y provisión de puestos de trabajo de los funcionarios de los Cuerpos Nacionales de Inspección, y la planificación de los recursos humanos y medios materiales del Sistema.

g) Proponer la adopción de acuerdos relativos a la aportación de recursos materiales, técnicos o económicos del Organismo Estatal y al perfeccionamiento profesional de los efectivos del Sistema, así como la adopción de criterios comunes sobre la estructura territorial y funcional del mismo, sin perjuicio de las competencias del Ministerio de Hacienda y Función Pública y de lo establecido en las respectivas leyes de presupuestos.

b) Aprobar los criterios para evaluar la eficacia y la calidad de los servicios del Organismo Estatal.

i) Conocer los informes, estadísticas y memorias a los que se refiere el art. 31.3.g) de la Ley 23/2015, de 21 de julio.

j) Aprobar, a propuesta del titular de la Dirección del Organismo Estatal, los criterios de distribución de los puestos de trabajo de personal inspector correspondientes a la estructura territorial del Organismo teniendo en cuenta, al menos, los indicadores de población activa, empleada y desempleada, siniestralidad laboral y extensión territorial.

Dichos criterios se tendrán en cuenta tanto para la determinación de la propuesta de ingreso en los cuerpos de inspección como en la fijación de las plazas en los procesos de provisión de puestos de trabajo en dichos cuerpos.

k) Aprobar su Reglamento de funcionamiento interno».

${ }_{10}$ Por Orden TMS/955/2018, de 17 de septiembre (BOE, 18) se designan miembros del Consejo Rector del Organismo Autónomo Organismo Estatal Inspección de Trabajo y Seguridad Social. 
Quizá en el futuro estas funciones podrían ser ampliadas con alguna más - por ejemplo, el ejercicio de la potestad sancionadora en las materias del orden social, que es, no hay que olvidarlo, una de las consecuencias, y quizá la más importante, de las actuaciones inspectoras-. Dado que está pendiente de desarrollo reglamentario el art. 48 del Texto Refundido de la Ley de Infracciones y Sanciones del Orden Social aprobado por Real Decreto Legislativo 5/2000, de 4 de agosto, que regula esta materia, podría haber ocasión para atribuir al Consejo Rector el ejercicio de la potestad sancionadora respecto de determinado tipo de infracciones del orden social, bien por revestir una especial entidad económica o por tener alcance supraautonómico, cuestión esta última que no está bien resuelta cuando se trata de infracciones sociales cuya sanción corresponde imponer a las Comunidades Autónomas (art. 149.1.7. ${ }^{\circ} \mathrm{CE}$ ), pero que afectan a empresas con centros de trabajo radicados en más de una de ellas. Teniendo en cuenta que en el Consejo Rector están representadas todas las Comunidades Autónomas, en estos casos parece que sería oportuno que el ejercicio de la potestad sancionadora para este tipo de infracciones recayera en el propio Consejo Rector. Y dicho esto se podría también atribuir al Consejo Rector el ejercicio de la potestad sancionadora respecto a infracciones sociales de competencia estatal por afectar al régimen económico de la Seguridad Social (art. 149.1.18. ${ }^{a}$ CE) y que actualmente deben ser ejercidas, en función de la cuantía de las sanciones propuestas, por la persona titular del Ministerio de Trabajo, Migraciones y Seguridad Social o incluso por el Consejo de Ministros, lo que se presenta como algo ciertamente extraño y disfuncional.

\section{B. El director}

El director es el principal órgano ejecutivo del Organismo, a quien se atribuye la representación y gestión del mismo, así como las funciones propias de la autoridad central de la Inspección de Trabajo y Seguridad Social de acuerdo con lo establecido en el Convenio número 81 de la Organización Internacional del Trabajo.

El director del Organismo sustituye a la figura del director general de la Inspección de Trabajo y Seguridad Social, órgano del Ministerio de Trabajo, Migraciones y Seguridad Social que queda suprimido por la nueva regulación ${ }^{11}$.

${ }^{11}$ Disposición final primera del Real Decreto 192/2018, de 6 de abril. 
Las funciones del director se especifican prolijamente tanto en la LOSITSS como en los Estatutos ${ }^{12}$. Un análisis detallado de estas funcio-

12 «Art. 8.3. Estatutos:

Con carácter específico, corresponden al titular de la Dirección las siguientes funciones:

a) La dirección, organización, gestión y coordinación del funcionamiento del Organismo Estatal.

b) Ejercer la representación del Sistema español de Inspección de Trabajo y Seguridad Social en las instituciones y en foros internacionales, así como de la Unión Europea, sin perjuicio de la participación de las Comunidades Autónomas en la forma que se determine.

c) Proponer proyectos de normas legales y reglamentarias en materias relacionadas con la Inspección de Trabajo y Seguridad Social

d) Definir los planes y programas generales de actuación de la Inspección de Trabajo y Seguridad Social, sin perjuicio de la participación de las Comunidades Autónomas.

e) Autorizar la adscripción a la Oficina Nacional de Lucha contra el Fraude de las empresas que deban ser objeto de comprobación e investigación por la Dirección Especial de Inspección, de acuerdo con los planes y programas generales que se establezcan.

f) La dirección de las funciones de alta inspección del Estado en el orden social de acuerdo con lo establecido en los Estatutos de Autonomía, en la forma establecida en la disposición adicional novena de la Ley 23/2015, de 21 de julio.

g) Celebrar o promover, en su caso, la celebración de convenios con las Comunidades Autónomas o con otras instituciones del Estado en materias competencia del Organismo Estatal y firmar los mismos cuando no corresponda a una autoridad superior.

b) Ostentar la Secretaría del Consejo Rector y presidir la Comisión Permanente del Consejo Rector.

i) Presidir el Consejo General del Organismo.

j) Elaborar y publicar informes, estadísticas y memorias sobre la actuación del Sistema de Inspección de Trabajo y Seguridad Social cuando se refieran al ámbito general del territorio del Estado o tengan por destinatario instituciones del Estado u órganos supranacionales o internacionales, así como su presentación a las instituciones de dichos ámbitos, y elaborar la memoria anual y los informes periódicos exigidos por los Convenios y Recomendaciones de la Organización Internacional del Trabajo sobre la Inspección de Trabajo y Seguridad Social, y los relativos al Comité de Altos Responsables de la Inspección de Trabajo de la Unión Europea.

k) Ostentar la jefatura del personal y organizar y gestionar los recursos humanos y medios materiales y económicos del Organismo Estatal, especialmente en materia de indicadores para la medición de la actividad individual y de los objetivos colectivos, selección, formación y movilidad, y funcionamiento de los servicios administrativos.

l) El impulso del desarrollo y la gestión de los sistemas de información necesarios para la planificación, programación, seguimiento y evaluación de las actuaciones inspectoras, así como de sistemas de análisis y sistemas de gestión del conocimiento dirigidos a facilitar la actuación inspectora, sin perjuicio de lo dispuesto en el art. 14. 3 de los presentes Estatutos.

$m$ ) Celebrar los contratos en nombre del Organismo en ejercicio de las facultades que le corresponden como órgano de contratación, conforme a lo establecido en el art. 100.2 de la Ley 40/2015, de 1 de octubre, y en la legislación sobre contratación del sector público.

n) La definición de los criterios técnicos y operativos para el desarrollo de la función inspectora, sin perjuicio de la participación de las Comunidades Autónomas.

o) El conocimiento y resolución de los expedientes y recursos señalados por la normativa aplicable, así como de aquellos otros incoados por la Inspección y que no correspondan expresamente a otras autoridades de las Administraciones Públicas. 
nes excede el propósito y la extensión de esta presentación, pero sí creo que debe ponerse énfasis en la figura del director como elemento clave en la nueva regulación para hacer real y efectiva la autonomía del Organismo con vistas a preservar y fortalecer la independencia y profesionalidad de las actuaciones que siempre han caracterizado a la Inspección de Trabajo y Seguridad Social y también para canalizar adecuadamente y cohonestar el ejercicio de sus funciones con las propias del Consejo Rector, así como estar en permanente disposición de contribuir a resolver las cuestiones y dificultades que puedan presentarse como consecuencia de la confluencia en el seno de dicho Consejo Rector de posiciones políticas diversas y, en ocasiones, opuestas.

\section{El Consejo General}

El Consejo General es el órgano de participación institucional del Organismo Estatal ITSS donde están representados los destinatarios naturales de las actuaciones inspectoras, esto es, los trabajadores y las empresas, a través de los sindicatos y las organizaciones empresariales que tienen la condición de más representativas.

Como tal órgano de participación institucional desarrolla funciones de información, audiencia y consulta sobre los asuntos relativos al Organismo

p) El conocimiento de las cuestiones que se planteen ante el Ministerio de Trabajo, Migraciones y Seguridad Social en relación con actuaciones de la Inspección de Trabajo y Seguridad Social, sin perjuicio de las competencias de las autoridades autonómicas.

q) La elaboración de la propuesta de presupuesto de ingresos y gastos del Organismo, así como el seguimiento de la ejecución presupuestaria y formular las cuentas anuales y rendirlas, una vez aprobadas, al Tribunal de Cuentas, por conducto de la Intervención General de la Administración del Estado.

r) Adoptar las instrucciones sobre guardias y organización de los servicios en las materias competencia de la Administración General del Estado en coordinación con las Comunidades Autónomas.

s) La propuesta y coordinación de los nombramientos de los peritos judiciales y del personal del Organismo en funciones de auxilio jurisdiccional, así como la coordinación de otras actuaciones de colaboración con los órganos judiciales.

t) La relación institucional con las autoridades competentes de las Comunidades Autónomas a efectos de asegurar la coherencia general del Sistema y promover la aplicación de los objetivos generales en su actuación.

u) La aprobación y compromiso de gastos, salvo los reservados por la Ley a la competencia del Gobierno, así como el reconocimiento de la obligación y el pago de las obligaciones a que se refiere el art. 74.2 de la Ley 47/2003, de 26 de noviembre.

v) Cualesquiera otras que le asigne la normativa vigente o le encomiende el Consejo Rector». 
Estatal de modo paralelo o en el nivel de conocimiento del Consejo Rector, del cual es, en cierto modo, antagonista. En este sentido se expresan los Estatutos al señalar que el Consejo General tendrá funciones de información, audiencia y consulta «en las materias de las que tenga conocimiento el Consejo Rector y respecto de las mismas» (art. 11).

El Consejo General también tiene un precedente inmediato, cual es la Comisión Consultiva Tripartita de la Inspección de Trabajo y Seguridad Social creada por la Orden TAS/3869/2006, de 20 de diciembre, que consecuentemente queda derogada por el Real Decreto 192/2018, de 6 de abril ${ }^{13}$.

Como la anterior Comisión Consultiva Tripartita, así también el Consejo General tiene una composición paritaria a tres, con ocho participantes de cada una de las representaciones: ocho por los sindicatos, ocho por las organizaciones empresariales y otros ocho por la Administración, con la novedad, en este caso, de que los representantes de la Administración se dividen en dos grupos a razón de cuatro por la Administración General del Estado y cuatro por las Comunidades Autónomas, dada la composición bifronte del Consejo Rector ${ }^{14}$.

El Consejo General estará presidido por el director del Organismo, contando con tres vicepresidencias que recaen: una, en un miembro del Consejo Rector designado por las Comunidades Autónomas y las otras dos, en los otros dos grupos de representación (sindicatos y organizaciones empresariales).

Al igual que el Consejo Rector, el Consejo General cuenta con unas normas de funcionamiento en los Estatutos y un régimen de adopción de acuerdos, a completar en su caso por su reglamento de funcionamiento interno, si bien puede indicarse que, dada la idiosincrasia del Consejo General y las características eminentemente consultivas y de asesoramiento propias del mismo, la posición que exprese el Consejo General respecto de los asuntos que se sometan a su consideración será, por regla general, la posición de cada una de las partes que lo componen, en especial la de los sindicatos y organizaciones empresariales, por lo que, en principio, puede parecer extraña la previsión de unas normas sobre régimen de adopción de acuerdos que quizá se han adoptado por emulación respecto de las mismas normas del Consejo Rector.

${ }_{13}$ Disposición derogatoria única, 2

14 Por Orden TMS/973/2018, de 20 de septiembre (BOE, 24) se designan miembros del Consejo General del Organismo Autónomo Organismo Estatal Inspección de Trabajo y Seguridad Social. 


\section{La estructura administrativa central del OEITSS}

El capítulo III de los Estatutos del OEITSS se refiere a la estructura administrativa central, el núcleo operativo del Organismo, como hemos indicado ya. Esta estructura se asienta sobre cuatro pilares básicos que son: la Oficina Nacional de Lucha contra el Fraude, la Subdirección General para la Coordinación de la Inspección del Sistema de Relaciones Laborales, la Subdirección General de Relaciones Institucionales y Asistencia Técnica, y la Secretaría General. Además, se integra en el Organismo la preexistente Escuela de la Inspección de Trabajo y Seguridad Social y se añade una Junta Consultiva como órgano de asesoramiento al director del Organismo.

Sin que sea posible por razones de espacio realizar un análisis exhaustivo de las funciones y cometido de estas unidades, sí creo que conviene dar unas breves pinceladas sobre la significación y alcance de estas unidades en la nueva regulación. Todas ellas provienen de la extinta Dirección General de la Inspección de Trabajo y Seguridad Social ${ }^{15}$, lo que garantiza la necesaria continuidad en el funcionamiento del Sistema de Inspección.

La Oficina Nacional de Lucha contra el Fraude, unidad ya mencionada por la LOSITSS ${ }^{16}$ como órgano encargado del impulso y coordinación de la aplicación de las medidas de lucha contra el trabajo no declarado, el empleo irregular, el fraude a la Seguridad Social y cuantas otras se determinen, así como de su coordinación e integración con el conjunto de la actuación inspectora, es una de las dos unidades temáticas de la estructura central del OEITSS (la otra es la Subdirección General para la Coordinación de la Inspección del Sistema de Relaciones Laborales a que nos referiremos después). Hay que aclarar que la Oficina como tal no es un órgano con funciones inspectoras, sino un órgano de análisis, planificación y programación de actuaciones inspectoras (a realizar, por tanto, por las unidades territoriales de Inspección a que nos referiremos después) sobre todas aquellas conductas relacionadas con el fraude. También hay que aclarar que el término fraude debe ser concebido en sentido amplio y la motivación para combatirlo es eminentemente social y no meramente recauda-

15 La Orden ESS/471/2018, de 26 de abril, por la que se adecuan los nombramientos de los titulares de las unidades con nivel orgánico de Subdirección General del Organismo Estatal Inspección de Trabajo y Seguridad Social (BOE, 9 de mayo de 2018), alude a la correspondencia de estas unidades con las anteriores.

${ }_{16}$ Disposición adicional segunda, 1. 
toria ${ }^{17}$. Es comúnmente aceptado, además, que los fenómenos del fraude son múltiples y mutantes: las formas del fraude en el trabajo no se limitan al puro y simple ocultamiento (trabajo no declarado en sentido estricto), sino que comprenden también la declaración de trabajo en cuantía inferior al efectivamente realizado (trabajo insuficientemente declarado) o el trabajo falsamente declarado, donde se incluye el fenómeno de los falsos autónomos, desde sus más burdas presentaciones a las más artificiosas, las cuales, al socaire de potentes estructuras tecnológicas y financieras, tratan de negar el fenómeno del trabajo por cuenta ajena por el debilitamiento de la nota de la dependencia propia de la relación laboral, pero obviando la abismal diferencia de posición entre la persona que realiza el trabajo y el titular de la colosal infraestructura en cuyo seno se presta; elemento este (la desigualdad real entre quien presta el trabajo y el que lo recibe) que propició el surgimiento de la figura del contrato de trabajo como evolución del tradicional arrendamiento del servicio proveniente del Derecho romano, al que parece nos retrotraen estas formas de trabajo que nos presentan ahora como el no va más de lo moderno. También los modos del fraude mutan y se adaptan. El fraude es irredimible: cada vez que se pone cerco a determinadas conductas vulneradoras de derechos surgen otras parecidas en las que se percibe un cambio en las formas, pero no en el espíritu.

La Inspección de Trabajo y Seguridad Social es la institución que está en la vanguardia de la respuesta de los poderes públicos a las diversas formas del fraude en el trabajo. La Oficina Nacional de Lucha contra el Fraude, como órgano del OEITSS, tiene como principal misión la observación de las distintas conductas infractoras; el desvelamiento de los patrones de estas conductas; el análisis de la información, para lo que cuenta ya con potentes medios de procesamiento de datos que deben ser mejorados y reforzados, y la planificación y, en su caso, programación de las actuaciones inspectoras, en consonancia con los planes y programas generales de

${ }_{17}$ Es destacable que la propia LOSITSS (exposición de motivos) se refiera al fraude de esta manera: «El fraude no solo supone una indebida detracción de recursos del Sistema de Seguridad Social: el fraude va ligado, la mayoría de las veces, a situaciones de explotación laboral en las que se niegan a los trabajadores los más elementales derechos, el más importante de ellos es el reconocimiento de su condición de trabajadores, lo que sucede cuando se los mantiene en un contexto de trabajo no declarado. De ahí que el mantenimiento de un sistema de Inspección de Trabajo y Seguridad Social integral, con los principios de unidad de función y acto inspector que ello conlleva y la especialización derivada del funcionamiento de la Oficina, permitirá un tratamiento del fraude de un modo global, sin menoscabo de la función social que siempre se ha aparejado a la actuación de la Inspección de Trabajo, lo que podría producirse si se limita la concepción de la Oficina a su faceta meramente recaudatoria y de represión de conductas ilícitas». 
actuación de la Inspección de Trabajo y Seguridad Social y las medidas y estrategias necesarias para su ejecución que se aprueban por el Consejo Rector del OEITSS. Sus funciones están definidas en el art. 16 de los Estatutos ${ }^{18}$. También es destacable que los Estatutos se refieran a la coordinación administrativa previendo fórmulas de auxilio y colaboración en materia de lucha contra el fraude. A ellos se refiere el art. 17 de los Estatutos.

La Subdirección General para la Coordinación de la Inspección del Sistema de Relaciones Laborales es la segunda unidad temática del OEITSS y se le asignan una serie de funciones definidas en los Estatutos (art. 18) ${ }^{19}$ que se aglutinan en torno a las materias propias de las compe-

18 «a) El análisis de los comportamientos y conductas fraudulentas en las materias de su competencia y la adopción de iniciativas para la formulación de estrategias generales para luchar precozmente contra los mismos.

b) Elaborar los programas y planes generales de actuación de la Inspección de Trabajo y Seguridad Social en materia de lucha contra el trabajo no declarado, el empleo irregular y el fraude a la Seguridad Social, así como su impulso y coordinación de tales programas entre los órganos centrales y territoriales de inspección.

c) Elaborar los planes de actuación de la Unidad Especial de Inspección en materia de formación profesional para el empleo competencia de la Administración General del Estado, integrándolos en los planes y programas territoriales de inspección, cuando así haya sido aprobado por la Comisión Operativa Autonómica de la Inspección de Trabajo y Seguridad Social.

d) La definición de métodos de trabajo y procedimientos de investigación y comprobación inspectora en materia de lucha contra el fraude.

e) Ejercer las competencias en materia de procedimientos sancionadores y expedientes liquidatorios por cuotas de la Seguridad Social y otros conceptos de recaudación conjunta iniciados por la Inspección de Trabajo y Seguridad Social, de acuerdo con lo dispuesto en la normativa específica en la materia.

f) Establecer y desarrollar los mecanismos de cooperación y colaboración necesarios con los Centros Directivos, Entidades Gestoras, Servicios Comunes y Organismos Autónomos del Ministerio de Trabajo, Migraciones y Seguridad Social.

g) Asumir, en el ámbito de las competencias de la Inspección de Trabajo y Seguridad Social, las funciones de comunicación, coordinación y colaboración en materia de lucha contra el fraude con otros Departamentos de la Administración General del Estado, con las Comunidades Autónomas u otras Administraciones Públicas.

b) Coordinar las actuaciones con otros Departamentos o Administraciones Públicas que colaboran en la lucha contra el fraude en el marco de los correspondientes convenios.

i) El control y seguimiento de las actuaciones realizadas, así como proceder a su evaluación periódica sobre su grado de cumplimiento y eficacia.

j) Proponer al titular de la Dirección del Organismo Estatal la celebración de convenios de colaboración con otras Administraciones Públicas en materia de lucha contra el fraude.

k) Cuantas otras le sean específicamente atribuidas por el titular de la Dirección del Organismo».

19 «a) El análisis, en coordinación con las Comunidades Autónomas, de los comportamientos y conductas infractoras en las materias de su competencia, y la adopción de iniciativas para la formulación de estrategias generales para luchar precozmente contra los mismos, en el marco de los órganos de participación previstos en esta Ley.

b) La coordinación con las Comunidades Autónomas de la actuación inspectora sobre las materias laborales, de prevención de riesgos laborales y medidas de igualdad cuya com- 
tencias de las Comunidades Autónomas; por ello resulta obligado coordinar con el objeto de lograr la máxima coherencia y homogeneidad posible en las actuaciones de la Inspección de Trabajo y Seguridad Social en el conjunto del territorio español.

La Subdirección General de Relaciones Institucionales y Asistencia Técnica es una unidad de asistencia jurídica y técnica que actúa transversalmente en todas las materias objeto de actuación inspectora y se encarga de las relaciones institucionales tanto con las Comunidades Autónomas como en el ámbito internacional. Sus funciones están definidas en el art. 19 de los Estatutos ${ }^{20}$.

petencia de ejecución corresponda a las Comunidades Autónomas o que hayan podido encomendarse a la Inspección mediante convenios de colaboración.

c) La elaboración, en colaboración con las Comunidades Autónomas, de planes y programas de alcance general en las materias a que se refiere la letra anterior, así como el seguimiento y evaluación de los resultados alcanzados.

d) La definición de métodos de trabajo y procedimientos de investigación y comprobación inspectora en su ámbito material de competencias en colaboración con las Comunidades Autónomas.

e) La coordinación de actuaciones con otros órganos del Departamento y las relaciones con otros organismos de la Administración General del Estado en relación con las materias indicadas en las letras anteriores, en particular en los programas de actuación para combatir la siniestralidad laboral.

f) La colaboración con la Fundación para la Prevención de Riesgos Laborales a efectos de lograr un mejor cumplimiento de los fines de la Fundación.

g) Cuantas otras funciones pueda encomendarle el titular de la Dirección del Organismo Estatal».

20 «a) La coordinación de la relación institucional, en particular con las Comunidades Autónomas.

b) La asistencia técnica a la actuación inspectora a través de la propuesta de instrucciones, de la propuesta de criterios técnicos y operativos y de consultas, en coordinación con las autoridades competentes por razón de la materia.

c) La elaboración de proyectos de disposiciones de carácter general relativas al Sistema de Inspección de Trabajo y Seguridad Social.

d) La preparación de las convocatorias de las pruebas selectivas para el acceso a los Cuerpos del Sistema, así como de los programas con arreglo a los cuales se han de ejecutar dichas pruebas.

e) El establecimiento, seguimiento y control de los indicadores de eficiencia y eficacia de la actuación inspectora y la supervisión del funcionamiento de las distintas unidades mediante un procedimiento de auditoría interna.

f) La coordinación o ejecución, en su caso, de actuaciones relativas a las relaciones internacionales, así como la recepción y remisión de la información relativa a la asistencia mutua internacional derivada de actuaciones inspectoras en materias competencia del Sistema de Inspección.

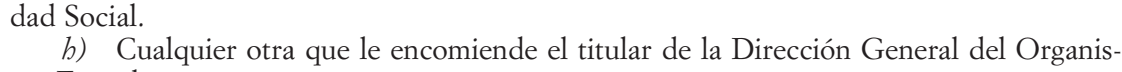
mo Estatal». 
La Secretaría General es la unidad que gestiona los servicios generales del OEITSS: personal, medios materiales (en especial los tecnológicos), gestión económica y presupuestaria ${ }^{21}$. Es en esta unidad donde se visualiza en sentido práctico la autonomía de gestión que es predicable de los organismos autónomos (personalidad jurídica pública diferenciada, patrimonio y tesorería propios, así como autonomía de gestión y plena capacidad jurídica y de obrar), lo que sin duda exigirá la necesidad de adecuación y refuerzo de su estructura con objeto de que pueda desempeñar con efectividad su misión. Dicha unidad también necesitará de una adecuada coordinación con la Subsecretaría del Ministerio de adscripción, el Ministerio de Trabajo, Migraciones y Seguridad Social, dado que, por mor de lo dispuesto en el art. 14 de los Estatutos, una serie de servicios comunes han de ser objeto de gestión compartida, lo cual ya estaba previsto en la LOSITSS (disposición adicional primera, 2) y en el art. 68 de la Ley 40/2015, de 1 de octubre, de Régimen Jurídico del Sector Público.

La Escuela de la Inspección de Trabajo y Seguridad Social es la unidad de formación y estudios del Sistema de Inspección desde su creación en el año 2009. Ya disponía de regulación propia a través del Real Decreto $1223 / 2009$, de 17 de julio, que expresamente se mantiene en los Estatutos del Organismo (art. 21).

La estructura central del OEISS se completa con una Junta Consultiva como órgano de asesoramiento del director y compuesta por los directores territoriales y otros funcionarios del Sistema que se convoquen a las reu-

$21 \ll$ Art. 20. Estatutos:

Corresponde a la Secretaría General el desarrollo de las siguientes funciones:

a) La gestión y administración de los recursos humanos del Organismo Estatal, la elaboración de propuestas de relaciones de puestos de trabajo, la tramitación de los procesos para la cobertura de puestos de trabajo, la acción social y la prevención de riesgos laborales.

b) La planificación y gestión de los medios materiales y económicos necesarios para el desarrollo de las funciones encomendadas al organismo autónomo Organismo Estatal Inspección de Trabajo y Seguridad Social.

c) La elaboración del anteproyecto de presupuesto del Organismo Estatal, así como el seguimiento de la ejecución presupuestaria y la tramitación, en su caso, de modificaciones presupuestarias y su gestión económica, financiera y contable.

d) La gestión de los ingresos y gastos, la realización de los cobros y pagos, y la gestión de la tesorería.

e) La custodia, administración, conservación y mantenimiento de los bienes inmuebles, adscritos o en propiedad, del Organismo Estatal y la tramitación de los expedientes de adscripción y desadscripción.

f) La seguridad, régimen interior, asuntos generales y la coordinación e inspección de las unidades, instalaciones y servicios del Organismo Estatal.

g) Cualquier otra que le encomiende el titular de la Dirección del Organismo». 
niones. Por su naturaleza no es un órgano de funcionamiento permanente. Su importancia radica en la cantidad y calidad del conocimiento que atesoran los directores territoriales sobre la realidad laboral de su Comunidad Autónoma que, puesta en conjunto, ofrece un inmenso valor para formar el criterio del OEITSS sobre los múltiples aspectos que integran el quehacer de la Inspección.

\section{La estructura administrativa territorial del OEITSS}

Las unidades inspectoras que desempeñan las funciones de inspección activa en las empresas son las integrantes de la estructura territorial. Se refiere a ella el capítulo IV de los Estatutos. La estructura territorial del OEITSS se divide básicamente en la Dirección Especial, con competencia en todo el territorio español, y las Direcciones Territoriales en las Comunidades Autónomas. Las Direcciones Territoriales disponen, además, de Inspecciones Provinciales en las Comunidades Autónomas pluriprovinciales.

La Dirección Especial de Inspección es la unidad del OEITSS con funciones inspectoras que actúa en la totalidad del territorio español. Esta unidad ya existía en la configuración anterior de la Inspección de Trabajo y Seguridad Social. La principal novedad que presenta la Dirección Especial del OEITSS respecto de la anterior es que ahora va a tener competencias en todos los ámbitos de actuación de la Inspección, según el art. 12.1 LOSITSS $^{22}$, y no solo respecto de ámbitos restringidos, como sucedía con la regulación anterior en que solo actuaba en aquellas materias de competencia de la Administración General del Estado cuando la actuación era de ámbito territorial supraautonómico ${ }^{23}$. Por esta circunstancia, la Dirección Especial es quizá la unidad operativa del OEITSS donde mejor cristaliza el principio ordenador de la LOSITSS de concepción única e integral del Sistema y funcionamiento cohesionado del mismo.

22 «Art. 12.1.a) sistema de relaciones laborales; $b$ ) prevención de riesgos laborales; $c$ ) sistema de la Seguridad Social; $d$ ) empleo; $e$ ) migraciones; $f$ ) cooperativas y otras fórmulas de economía social, así como a las condiciones de constitución de sociedades laborales, salvo que la respectiva legislación autonómica disponga lo contrario en su ámbito de aplicación; g) cualesquiera otros ámbitos cuya vigilancia se encomiende legalmente a la Inspección de Trabajo y Seguridad Social».

${ }^{23}$ Art. 58 (derogado) del Reglamento de Organización y Funcionamiento de la Inspección de Trabajo y Seguridad Social aprobado por el Real Decreto 138/2000, de 4 de febrero. 
El principio general de actuación de la Dirección Especial es el de coordinación de las actuaciones inspectoras sobre empresas, sectores o situaciones que excedan del ámbito territorial de una Comunidad Autónoma, así como las que versen sobre los entes, organismos y entidades que forman parte del sector público estatal, lo que implica que el desarrollo directo de las actuaciones se realizará por los funcionarios destinados en las unidades territoriales de Inspección correspondientes. En este sentido, el art. 12.4 de los Estatutos es explícito al señalar que «la Dirección Especial, en los supuestos a los que se refiere este artículo, coordinará, en el ámbito operativo, las actuaciones de las Direcciones Territoriales e Inspecciones Provinciales del Organismo Estatal y asumirá su dirección técnica bajo el principio de unidad de acción y de criterio» ${ }^{24}$.

No obstante, en determinados ámbitos de actuaciones, coincidentes con las materias cuya competencia corresponde a la Administración General del Estado ${ }^{25}$, la Dirección Especial puede asumir, junto a la coordinación, el desarrollo directo de las actuaciones inspectoras.

\footnotetext{
${ }^{24}$ La coordinación de las actuaciones de ámbito supraautonómico cuando están afectadas Comunidades Autónomas que hayan recibido el traspaso de servicios en materia de función pública inspectora exigía un régimen especial indicado en el art. 25 de los Estatutos: «En las Comunidades Autónomas que hayan recibido el traspaso de funciones y servicios en materia de función pública inspectora, la Dirección Especial de Inspección se coordinará, sobre el mismo principio de unidad de acción y de criterio, con los servicios de inspección adscritos orgánicamente a dichas Comunidades. Los términos y el alcance del ejercicio de dicha coordinación se establecerán en el órgano de cooperación multilateral al que se refiere el apartado 2 de la disposición adicional octava de la Ley 23/2015, de 21 de julio».

25 «Art. 24.2. Estatutos:

2. En el ámbito de las competencias de la Administración General del Estado, la Dirección Especial podrá desempeñar, junto a la coordinación, la dirección o el directo desarrollo, en su caso, de las siguientes actuaciones:

a) Actuaciones inspectoras sobre empresas, sectores o situaciones que excedan del ámbito territorial de una Comunidad Autónoma.

b) Actuaciones inspectoras sobre los entes, organismos y entidades que forman parte del sector público estatal.

c) La inspección de las mutuas colaboradoras con la Seguridad Social y las actuaciones inspectoras relativas a la colaboración voluntaria de las empresas en la gestión de la Seguridad Social.

d) La inspección de centros de la Administración del Estado, en cuanto a sus sedes centrales o cuando la actuación exceda del ámbito autonómico.

e) Las actuaciones inspectoras que correspondan a programas generales, a objetivos señalados por órganos de la Unión Europea en la esfera de su competencia o los que se acuerden respecto de materias de competencia compartida, sin perjuicio de la competencia que corresponda a las Comunidades Autónomas.

f) La emisión de los informes que le solicite la Administración General del Estado.

g) Las actuaciones que le sean encomendadas por los órganos de dirección del Organismo Estatal en la esfera de su competencia».
} 
La intervención de la Dirección Especial, al asumir un papel de coordinación de las actuaciones inspectoras en las empresas de ámbito supraautonómico cuando se requiera la multiplicidad de intervención en los centros de trabajo radicados en distintas Comunidades Autónomas, permite la garantía de una respuesta uniforme y coherente de la Inspección para la empresa en su conjunto, sin perjuicio de que el desarrollo directo de las actuaciones, previamente coordinadas por la Dirección Especial, esto es, la interlocución con las empresas y los trabajadores mediante visitas de inspección u otras comprobaciones y, en su caso, la adopción de las medidas resultantes (requerimientos, advertencias o actas de infracción, entre otras), se practiquen por las unidades territoriales de Inspección (Direcciones Territoriales o Inspecciones Provinciales).

Los supuestos en los que la Dirección Especial puede asumir el desarrollo directo de las actuaciones se limita a las comprobaciones que versan sobre materias cuya competencia está atribuida al Estado y corresponde, por tanto, a este el ejercicio de la potestad sancionadora respecto de dichas actuaciones. En los casos de materias del orden social cuya competencia recae en las Comunidades Autónomas, la Dirección Especial no puede desarrollar directamente las actuaciones de inspección que pudieran dar lugar a la imposición de sanciones del orden social, precisamente porque el ejercicio de la potestad sancionadora recae en las Comunidades Autónomas, siendo que la Dirección Especial no está vinculada directamente con ninguna de ellas por su propia naturaleza. De ahí que, en estos casos, sean las unidades territoriales del nivel de Comunidad Autónoma o provincial las que deban desarrollar directamente las actuaciones y no la Dirección Especial, pues son aquellas y no esta las que dependen funcionalmente de las Comunidades Autónomas (también orgánicamente en los casos de Cataluña y el País Vasco).

\section{A. Las Direcciones Territoriales}

Las Direcciones Territoriales del Organismo desarrollan las actuaciones inspectoras de su competencia y dirigen y coordinan la actuación de las Inspecciones Provinciales, dentro del territorio de cada Comunidad Autónoma, con la estructura y los medios precisos para el correcto desarrollo de su función (art. 27.1 de los Estatutos del OEITSS). Estas unidades preexistían también a la actual regulación, si bien ahora se ven reforzadas en cuanto van a constituir una unidad de actuación inspectora de ámbito 
autonómico y no solamente provincial. Estas actuaciones podrán ser realizadas no solo por personal inspector con destino propio en las Direcciones Territoriales, sino también por personal habilitado temporalmente en las inspecciones provinciales integradas en la Dirección Territorial ${ }^{26}$, lo que dota de mayor flexibilidad y permite un mejor aprovechamiento de los recursos del OEITSS.

Los directores territoriales del Organismo Estatal que están al frente de cada una de estas unidades son también elementos clave de la arquitectura del Sistema de Inspección, dado su significativo papel vertebrador de las Administraciones Públicas que lo integran. Así, deben ser designados con la intervención de las Comunidades Autónomas en los términos previstos en el convenio de colaboración, lo que implica que se haga normalmente mediante acuerdo y se garantiza también la doble dependencia funcional de la Administración General del Estado o de la Administración autonómica correspondiente, en atención a la competencia material objeto de las actuaciones inspectoras en las que intervengan. A tal efecto despliegan una importante labor de interlocución con los agentes sociales en el territorio de la Comunidad Autónoma y de relación institucional con las autoridades autonómicas y estatales del territorio, coordinando la comisión operativa autonómica a que alude el art. 34 LOSITSS.

\section{B. Las Inspecciones Provinciales}

Las Inspecciones Provinciales de Trabajo y Seguridad Social son las unidades territoriales de Inspección con más tradición y raigambre, ya que existían desde la fecha de creación de la Inspección de Trabajo allá por el año 1906, respondiendo a la división territorial de España de 1833 formulada por el entonces ministro de Fomento, Javier de Burgos, y que se mantiene vigente. Los Estatutos del OEITSS, no obstante, señalan la posibilidad de organizar el despliegue periférico del Organismo Estatal sobre una base diferente como consecuencia de peculiaridades del territorio de la Comunidad Autónoma (art. 29.2).

Las Inspecciones Provinciales son las unidades del OEITSS que asumen el ejercicio de la función inspectora en el territorio de la provincia.

${ }^{26}$ «Art. 27.5. Estatutos: se podrá habilitar a personal inspector destinado en las inspecciones provinciales para desarrollar actuaciones de ámbito autonómico o pluriprovincial bajo la dirección del titular de la Dirección Territorial». 
Normalmente la sede de la Inspección Provincial se encuentra en la capital de la provincia, salvo en Pontevedra, donde la inspección se encuentra en Vigo. En Asturias y en Cádiz hay dos sedes de Inspección: Oviedo y Gijón en Asturias, y Cádiz y Algeciras en Cádiz. En las Comunidades Autónomas de las Islas Baleares y de las Islas Canarias, los propios Estatutos prevén que la organización y dotación de las Inspecciones podrá responder a las peculiaridades derivadas de la insularidad. Por ello, en las islas Baleares hay sede de la Inspección Provincial tanto en Palma de Mallorca como en Ibiza y Menorca. En las islas Canarias hay sede de la Inspección en las capitales de cada provincia, Santa Cruz de Tenerife y Las Palmas de Gran Canaria, y también en Arrecife (isla de Lanzarote), en Puerto del Rosario (Fuerteventura) y en Santa Cruz de la Palma (isla de La Palma).

Cada Inspección Provincial es dirigida y organizada por un jefe de inspección cuyas funciones se coordinan por el titular de la Dirección Territorial. En las Comunidades Autónomas uniprovinciales el director territorial asume las funciones de jefe de inspección.

\section{La estructura administrativa funcional del OEITSS}

De modo paralelo a la estructura territorial del OEITSS existe una estructura funcional que permite una cierta división del trabajo para el mejor cumplimiento de los fines y cometidos del Organismo. En esta línea, al tiempo que la LOSITSS proclama el principio de unidad de actuación inspectora en todas las materias del orden social, también incluye los criterios de especialización funcional y de actuación programada ${ }^{27}$.

La complejidad de los ámbitos de actuación funcional de la ITSS y la necesidad de prestar una especial atención a cuestiones específicas exige la creación de unidades especializadas que pueden desarrollar con mayor efectividad las actuaciones de planificación propia de la ITSS, en contraposición con las actuaciones rogadas (denuncias, peticiones de informes provenientes de Juzgados u otras autoridades, etc.).

Los Estatutos del OEITSS prevén la existencia de unidades especializadas de acción inspectora en todas las unidades territoriales (Dirección Especial, Direcciones Territoriales e Inspecciones Provinciales de Trabajo y Seguridad Social), si bien su constitución y composición responderá a las circunstancias de cada territorio, según lo que establezcan las relaciones de

27 Art. 2.c) LOSITSS. 
puestos de trabajo y, en su caso, mediante los convenios de colaboración con las Comunidades Autónomas, según el art. 25.2 LOSITSS ${ }^{28}$.

Las áreas funcionales de las unidades especializadas son las de Seguridad y Salud Laboral, Relaciones Laborales y Medidas de Igualdad, y también las propias de la Oficina Nacional de Lucha contra el Fraude. Estas unidades desarrollan actuaciones inspectoras que versan singularmente sobre materias de su área funcional de especialización, lo que no impide desarrollar otro tipo de actuaciones sobre otras áreas funcionales cuando las circunstancias del caso lo requieran ${ }^{29}$, salvaguardando así la compatibilidad del principio de unidad de función y acto inspector con el de especialización funcional.

Finalmente, los Estatutos del OEITSS se refieren a los equipos de inspección, que son la estructura básica de la acción inspectora en cuyo seno se desarrollan los principios de especialización y de unidad de función y de acto inspector ${ }^{30}$. Los equipos de inspección desarrollan las actuaciones individualizadas de inspección en las empresas y en los centros de trabajo. Se componen de varios funcionarios de Inspección, inspectores o subinspectores, al frente de los cuales existe un inspector de Trabajo y Seguridad Social. Cada equipo asume la ejecución de la programación de los servicios que correspondan a su ámbito, organizando y distribuyendo el trabajo entre sus miembros y el seguimiento de su ejecución y de la adecuación de las actuaciones practicadas, y desarrolla su cometido mediante la actuación individual o colectiva de sus inspectores y subinspectores.

\section{RÉGIMEN ECONÓMICO, PATRIMONIAL, DE CONTRATACIÓN Y DE RECURSOS HUMANOS}

La creación de un Organismo Autónomo para la Inspección de Trabajo y Seguridad Social obliga a la previsión de una infraestructura jurídica, patrimonial y financiera propia de la Administración institucional. Por ello los Estatutos dedican su capítulo $\mathrm{V}$ al régimen económico, patrimo-

28 Art. 31 de los Estatutos OEITSS.

29 Por ejemplo, resulta obvio que un inspector de Trabajo y Seguridad Social integrado en una Unidad Especializada de Seguridad y Salud Laboral podrá comprobar en el transcurso de una visita cuyo objeto es el de inspeccionar las condiciones de seguridad y salud en un centro de trabajo (una obra de construcción, por poner un caso) si los trabajadores se encuentran correctamente afiliados y en el alta en la Seguridad Social.

${ }^{30}$ Art. 33 de los Estatutos OEITSS. 
nial, de contratación y de recursos humanos del OEITSS. Los Estatutos remiten a la regulación general los distintos aspectos del régimen patrimonial (art. 36), el régimen presupuestario y de contratación (art. 37) o el control de la gestión económico-financiera (art. 38). En cuanto a los recursos financieros del OEITSS, también se detallan con toda su potencialidad $^{31}$, lo que no significa que actualmente estos recursos estén compuestos necesariamente de todas y cada una de las fuentes financieras que se detallan en los Estatutos, aunque sería deseable no solo por la necesidad de que el OEITSS cuente con una financiación suficiente que resulta imprescindible para el cumplimiento de sus fines, sino también por la necesidad de implicar en este terreno a todas las Administraciones Públicas titulares de las competencias materiales en las distintas actuaciones de la Inspección de Trabajo y Seguridad Social. Todo esto se verificará en los futuros escenarios presupuestarios, visto que actualmente, dado el reciente nacimiento del OEITSS a la vida pública, su financiación se realiza con cargo a los créditos presupuestarios de procedencia, de acuerdo con lo señalado en la disposición transitoria primera del Real Decreto 192/2018, de 6 de abril.

En cuanto al régimen relativo a los recursos humanos, los Estatutos lo remiten a la LOSITSS y al resto de la regulación general, esto es, al texto refundido de la Ley del Estatuto Básico del Empleado Público, aprobado por el Real Decreto Legislativo 5/2015, de 30 de octubre, y demás legislación sobre función pública inspectora, así como la Ley 47/2003, de 26 de noviembre, General Presupuestaria, y las respectivas leyes de presupuestos de cada ejercicio.

\footnotetext{
31 «Art. 35. Estatutos:

Los recursos económicos del Organismo Estatal estarán integrados por:

a) Las asignaciones que anualmente se establezcan con cargo a los Presupuestos Generales del Estado.

b) Las transferencias corrientes o de capital que, en virtud de convenios, encomiendas de gestión u otros instrumentos jurídicos, puedan acordarse con la Administración de la Seguridad Social, con las Administraciones de las Comunidades Autónomas o con otros órganos vinculados a la consecución de los objetivos establecidos en los correspondientes planes y programas de actuación inspectora o dirigidos a facilitar la realización de la actividad inspectora y al perfeccionamiento y especialización de los efectivos de la Inspección.

c) Los bienes y valores que constituyen su patrimonio.

d) Los productos y rentas de dicho patrimonio.

e) Las subvenciones, así como los ingresos que obtenga como consecuencia de conciertos o convenios con entes públicos.

f) Cualquier otro recurso no previsto en los apartados anteriores y que legítimamente pueda corresponderle».
} 
Por último, los Estatutos aluden a la tradicional doble dependencia de los funcionarios de los Cuerpos de Inspección ${ }^{32}$ respecto de la Administración estatal o autonómica que resulte competente por la materia objeto de inspección, lo que es connatural a la diversidad de las funciones que tienen encomendadas.

\section{CONCLUSIÓN}

El OEITSS supone un importante avance en la organización de la Inspección de Trabajo y Seguridad Social y refuerza el valor de esta institución, profundamente arraigada en el mundo de la empresa y del trabajo en España. Mantener y consolidar el Sistema de Inspección como instrumento de supervisión de toda la normativa del orden social, tan múltiple y diversa y, al mismo tiempo, tan intensamente entrelazada, es un desafío necesario que contribuye decisivamente a salvaguardar los derechos de los trabajadores, a la sostenibilidad de las pensiones y a garantizar el cumplimiento de las reglas del juego. Este desafío, que está en la génesis de la reforma del Sistema de Inspección iniciada por la LOSITSS y culminada por los Estatutos del OEITSS cuya presentación ahora finalizamos, requiere afrontarse con la confianza de todos y también con ambición y amplitud de miras, de modo que la reforma organizativa se acompañe de una voluntad real de reforma de la Inspección que conduzca efectivamente a alcanzar el fin perseguido de fortalecer la institución, fin que - no se olvide- no es fin en sí mismo, sino medio a su vez para el logro de los fines del Estado social, democrático y de Derecho que propugna nuestra Constitución.

${ }^{32}$ Los Cuerpos de Inspección (inspectores de Trabajo y Seguridad Social y subinspectores laborales) son Cuerpos Nacionales (art. 3.3 LOSITSS). 